\title{
LAS TEORÍAS DE LA ARGUMENTACIÓN EN EL TIEMPO I: LA ÉPOCA FUNDACIONAL
}

\author{
Argument theories during time I: The foundational time
}

Eduardo Risco del Valle*

Resumen

La historia de las teorías de la argumentación es, en términos generales, la historia de la evolución del uso de estructuras, procedimientos y recursos y, probablemente por eso mismo, subsisten en ella todavía algunos problemas no resueltos como la periodización, la conceptualización, los índices descriptores y si debemos hablar de varias teorías o de una sola construida con diversos aportes a lo largo de la tradición. En las páginas que siguen enunciamos estos problemas, proponemos una ampliación para uno de los esquemas clásicos de periodización y nos centramos fundamentalmente en presentar algunos elementos relevantes que permitan trazar una imagen del primero de estos períodos, conocido como "fundacional".

Palabras clave: Argumentación, Historia de las teorías de la argumentación, Problemas, Periodización.

\section{Abstract}

The history of argument theories is, in general, the history of the evolution of the use of structures, procedures and resources. Probably due to that, some unresolved problems such as periodization, conceptualization, descriptor indices, and whether we should talk about one or several theories built upon different contributions along the tradition, still remain. In this paper, we state these problems, propose an extension to one of the classical periodization schemes, and focus primarily on presenting some relevant elements in order to draw a picture of the first of these periods known as "foundational".

Key words: Argumentation, History of argument theories Problems, Periodization.

\section{INTRODUCCIÓN}

Este trabajo, que forma parte de una investigación mayor realizada en el marco del Proyecto MECESUP UCV0004 (UCO), pretende enunciar, de manera sucinta y problematizadora, una propuesta general de periodización del desarrollo de las teorías de la argumentación y, principalmente, trazar una imagen general de lo que hemos convenido en llamar período fundacional. Para conseguir estos fines, presentamos primero - y en estricto apego a las restricciones de espacio_-, de la manera más escueta posible, una revisión de los principales problemas involucrados en la cuestión de la periodización del desarrollo de las teorías de la argumentación, enunciando problemas que esperamos dilucidar en futuras contribuciones; en segundo lugar, y a fin de trazar una imagen del primero de los cortes cronológicos tratados (la época 
fundacional), ofrecemos una imagen de los principales aportes del período antes mencionado a la constitución de las teorías de la argumentación .

\section{LAS CUESTIONES VINCULADAS A LA PERIODIZACIÓN}

Cualquier intento por trazar un panorama del desarrollo histórico de las teorías de la argumentación se encontrará indefectiblemente con a lo menos cuatro problemas que en la literatura especializada aún podemos rotular como "por resolver", a saber: la periodización, la conceptualización, los índices descriptores más adecuados para caracterizar cada teoría y, finalmente (pero quizás el más complejo de todos), el problema de resolver si hablamos de una sola "macroteoría" construida con diversos aportes a lo largo de la tradición ${ }^{1}$ o de teorías diversas ${ }^{2}$.

La cuestión de la periodización parece ser la menos tratada y también respecto de ella no aparecen mayores disensiones en la literatura especializada, como no sean las diferencias que suscita la mayor o menor confusión terminológica entre los designadores "retórica" y "argumentación" y también "persuadir" y “convencer", que en unos casos aparecen en relación de sinonimia y, en otros, claramente diferenciados. La cuestión es relevante porque, si entendemos retórica y argumentación como términos sinónimos, será forzoso entonces afirmar que sus orígenes se remontan a una época que bordea el siglo VIII a de C., toda vez que los testimonios más antiguos de discursos construidos según una preceptiva estructural aparecen ya diseminados en las rapsodias más antiguas de la Iliada, lo que nos permitiría hablar de una teoría todavía más antigua que este texto. En cambio, si aceptamos que retórica y argumentación son cuerpos de conocimiento relacionados pero diferentes, entonces ya podremos contar con al menos un hito fundador: la composición del manual del maestro Korax de Siracusa, ocurrida con mucha probabilidad alrededor del año 450 a. de C. Esta segunda postura es la que adoptamos en este trabajo y, a partir de ella, nos resulta conveniente acoger, en primera instancia, la periodización en cuatro momentos que ofrecen Breton y Gauthier (2000), aunque ajustándola con nuestra propia disquisición al respecto debido al vacío de más o menos 20 siglos que estos autores admiten en su tratamiento del tema y que, a simple vista, parece imposible ${ }^{3}$. Para estos autores existirían cuatro períodos más o menos

\footnotetext{
${ }^{1}$ Postura que podría justificarse desde la perspectiva de que las distintas teorías parecen ocuparse de los mismos objetos siempre.

${ }^{2}$ Cuestión que, a su vez, podría resultar justificada fundamentalmente por el hecho de que lo que parece tomarse como criterio diferenciador es el uso de un concepto distinto de argumentación por cada una de ellas.

${ }^{3}$ Es posible que la razón de esta extraña omisión resida en que Breton y Gauthier parecen construir su periodización desde la lógica de la hegemonía ideológica y política del mundo grecolatino, fuera de ella, efectivamente, no es posible reconocer una vigencia "universalmente testificada" de los usos argumentativos, sin embargo, aun cuando este hecho pueda deberse a la ausencia de documentos históricos, también es posible probar que el ejercicio argumentativo (que efectivamente había abandonado los espacios públicos) encontró otros más específicos y menos visibles (la apologética cristiana entre ellos) en los que continuaron desarrollándose las teorías de la argumentación hasta su re-emergencia en 1958.
} 
claramente diferenciados en el desarrollo de las teorías de la argumentación, a saber: un período fundador, constituido por los trabajos de Korax, la hegemonía didáctica de los sofistas llegando hasta Platón; un período de madurez, que comprendería el apogeo aristotélico hasta el final de la república romana; un período de declive, que iría desde los comienzos del imperio romano hasta mediados del siglo $\mathrm{XX}, \mathrm{y}$, finalmente, un período de renacimiento, inaugurado con la publicación de la Nueva Retórica de Perelman y Olbrecht-Tyteca y que se prolongaría hasta nuestros días. Esta periodización, aunque no suficientemente justificada por los autores mencionados, nos parece — en general - adecuada y de sentido común, aunque, desde nuestra perspectiva, cabría el reconocimiento de dos períodos más dentro de lo que ellos rotulan como declive y que estarían constituidos tanto por el desarrollo de la argumentación apologética y su consecuencia lógica: la homilética cristiana, como por el período de auge de la escolástica medieval, asunto acerca del que volveremos más adelante, pero que por el momento resolveremos diciendo que, a nuestro juicio, son seis los períodos reconocibles en el desarrollo de las teorías de la argumentación: un período fundacional, originado con Korax, desarrollado por los sofistas y que gira fundamentalmente en torno al desarrollo de técnicas de persuasión; un período de madurez, centrado en la obra de Aristóteles y que se inaugura con la distinción —no siempre explícita - entre "persuadir" y "convencer", extendido hasta Quintiliano (aunque sería más propio decir que hasta la muerte de Cicerón); un período de declive, marcado fundamentalmente por el progresivo distanciamiento de la argumentación respecto de la retórica; un período de reconducción, representado por la asunción de la herencia argumentativa en el seno de la apologética cristiana; un período de surgimiento de la argumentación académica en el seno de la escolástica; y, finalmente, un período de refundación, inaugurado con Perelman y Toulmin y que se extiende hasta nuestros días. Sin embargo, es preciso subrayar que entre el período fundacional y el período de refundación ocurre un hecho muy importante y es la drástica reducción de las dimensiones del ámbito de uso y aplicación de las teorías de la argumentación, ya que estas, de ser un instrumento de uso cotidiano en la vida pública de los ciudadanos y vinculado al sostenimiento del mismísimo orden público democrático, pasan a ser un instrumento de uso más restringido en ámbitos sociales más privados y cada vez más vinculados a élites de conocimiento, proceso que alcanza su máxima expresión durante el período escolástico, en donde tiene exclusivo uso en el espacio del claustro universitario, protegido por la autonomía que la institución universitaria alcanza desde 1215.

La cuestión de la conceptualización, por su parte, parece más una cuestión definitoria que de conceptualización propiamente tal. En efecto, si hoy podemos hacer una distinción entre retórica y argumentación es porque, al perder relevancia la argumentación (en su período de declive), lo que sí logró asentarse en el nuevo contexto fue la preceptiva orientada a la estructuración y embellecimiento del discurso, ampliamente desarrollada ya en los "manuales patéticos" (centrados en 
técnicas para la movilización/manipulación de las pasiones del auditorio) de la sofística, de manera tal que, lo que originalmente era (o parecía) un mismo cuerpo de conocimientos, se separó en dos: una estilística, prontamente consumida por la ciencia literaria: la retórica; y una argumentación, entendida como ciencia de la composición de argumentos que fue paulatinamente relegada al terreno de la lógica matemática. Una distinción que, aunque empobreció el corpus de conocimientos originales y sus naturales mutuas relaciones, hoy nos permite decir que el contexto de origen e impulso de las teorías de la argumentación es la retórica o estilística clásica, de suyo anterior en varios siglos como lo hemos señalado más arriba. De esta manera, pues, y para los fines de este trabajo, entenderemos por retórica al estudio de las figuras de estilo y de las modalidades de expresión, en tanto que la argumentación será para nosotros el estudio de los procedimientos destinados a la composición de argumentos destinados a persuadir o convencer. Acciones estás últimas (persuadir y convencer) que hoy entendemos como apelación a la emocionalidad del auditor y apelación a la racionalidad del mismo, respectivamente, pero que en la tradición clásica aparecen habitualmente confundidas, aun cuando el propio Dionisio de Halicarnaso ${ }^{4}$ utiliza los verbos $\pi \imath \theta \alpha v \omega$ y $\pi \iota \sigma \tau o \omega^{5}$ para distinguirlas.

La cuestión de los índices descriptores para los distintos textos constituye, a su vez, un problema general que debe abordar la pragmalingüística cada vez que se asoma a los dominios de la argumentación, y esto, entre otras, por las siguientes razones: en primer lugar, porque la mayor parte de los estudios pragmalingǘsticos respecto de la argumentación provienen de la tradición analítica de la lingüística del discurso y esta ha ejercido su esfuerzo analítico fundamentalmente sobre corpus textuales monoconstruidos, en tanto que la argumentación implica el enfrentamiento de textos policonstruidos que no es posible abordar sin una asunción teórica del concepto de interacción, de manera que tales textos no han merecido todavía una adecuada atención de los analistas (baste como prueba de ello el hojear cualquier manual de análisis del discurso para comprobarlo), lo que lleva, finalmente, a un tratamiento de los corpora argumentativos con criterios propios de los corpora textuales monoconstruidos, todos ellos un tanto ajenos a la naturaleza interactiva de los mismos; y esto nos lleva, finalmente, a la necesidad de encontrar índices descriptivos más adecuados a la naturaleza argumentativa e interactiva de los textos que nos ocupan; un problema que, debido a la amplitud de las consideraciones teóricas que procederían, propondremos resolver siguiendo de momento los índices que la propia tradición clásica parece consagrar en los juicios que formulan sus autores acerca de otros retores y que bien podrían formularse del modo siguiente:

\footnotetext{
${ }^{4}$ Nuestro acceso a esta fuente se produce a través de una edición crítica realizada en 1826 por E. Gros con el título de: Examen critique del plus célèbres écrivains de la Grèce, par Denys d'Halicarnasse. Paris, Brunot-Labbe éditeur. Nois atenemos a los contenidos del primer tomo y, en adelante, referimos a esta obra todas las remisiones a Dionisio de Halicarnaso.

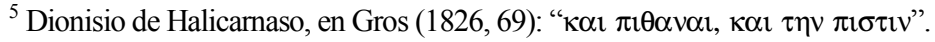


a) Respeto del orden canónico de estructuración y desarrollo del discurso argumentativo. Un hecho en el que en realidad no se insiste en la literatura clásica porque se da por supuesto que es la existencia misma de este tipo de discurso, enfatizándose muchísimo, sin embargo, la mayor o menor maestría que los distintos retores manifiestan en la construcción de las distintas partes y, así, Dionisio de Halicarnaso subraya que Lisias fue maestro en la composición del exordio, en tanto que Isócrates poseería una particular pericia en la disposición y distribución de las pruebas y otro tanto afirma de Iseo.

b) Establecimiento y sostenimiento de la cuestión del debate. Se trata de un aspecto tenido por fundamental en la construcción del discurso hasta nuestros días y consistente, fundamentalmente, en enunciar con claridad aquello acerca de lo que se discutirá: el objeto de la discusión; ya el propio Dionisio señala que "los retores aconsejan hacer dócil al auditorio y, para llegar a conseguirlo, exponer en pocas palabras el objeto del discurso a fin de que no resulte desconocido a los jueces" (82-83), y señala la pericia de Lisias para introducir claramente este objeto en el exordio.

c) Licitud y oportunidad de los argumentos empleados. Se trata de un índice estrechamente vinculado al anterior y que recubre dos aspectos fundamentales: en primer lugar, la evitación de recursos argumentales "tramposos" (conocidos habitualmente como "falacias") que resultarían ilícitos porque desvían el debate de la cuestión originalmente planteada $\mathrm{y}$, en segundo lugar, recubre también la "pertinencia" de los argumentos empleados, pertinencia medida por su acomodación a los fines de la discusión como al tipo de auditorio.

d) Claridad y pureza del estilo. Se trata de un índice que, aunque muy relevante en las evaluaciones que la tradición clásica hace de sus retores y discursos, hoy resulta menos considerado debido, probablemente, a que supone el arraigo del discurso en el contexto de una lengua particular y, para el caso que nos ocupa aquí, el Griego. En efecto, cuando Dionisio se refiere a este aspecto en Lisias, subraya que es un modelo perfecto del dialecto ático porque "presenta los pensamientos mediante palabras tomadas en su acepción propia y usual... y... rara vez se encuentra en sus discursos expresiones figuradas" (18).

\section{EL PERIODO FUNDACIONAL O EL APORTE DE LA SOFÍSTICA}

Es probable que el primer y principal aporte de este período al desarrollo de las teorías de la argumentación deba situarse en la introducción de la idea orden o estructura del discurso argumentativo, idea presidida por la convicción de que un discurso ordenado puede alcanzar de mejor manera los fines de la argumentación, a saber: persuasión y/o convicción del auditorio respecto de la cuestión defendida. Sin detrimento de lo anterior, Plantin (8-11), en su consideración indirecta de este período (él no parece tener en cuenta la periodización de la que hemos dado cuenta más arriba), indica otros cuatro grandes aportes, los que él rotula como: la Antifonía, la 
Paradoja, lo Probable y la Dialéctica; de tal manera que un primer catálogo de estos aportes-aprendizajes podría resultar compuesto del modo siguiente:

- Introducción de la idea de "orden del discurso argumetativo"

- Establecimiento de la Antifonía como espacio natural de ocurrencia

- Establecimiento de la Dialéctica como procedimiento

- Legitimación de la Paradoja como posibilidad contraargumentativa

- Establecimiento de la Probabilidad como forma argumental.

Evidentemente, un catálogo semejante solo es posibilitado en el marco de un contexto social y discursivo que podríamos llamar (a falta de un término más adecuado) Sofística.

a) La introducción de la idea de orden del discurso

Más allá de la discusión respecto de si fue Homero quien inauguró o plasmó la existencia de una costumbre de construir discursos argumentativos ${ }^{6}$ como los conocemos hoy, lo cierto es que tanto la literatura clásica como las investigaciones contemporáneas al respecto parecen coincidir en que la primera vez que una obra escrita tiene en cuenta el establecimiento de una preceptiva organizacional del discurso argumentativo es el manual del maestro $\operatorname{Korax}^{7}$ de Siracusa, quien probablemente redactó su obra hacia el año 460 a. de C. y a la que hoy accedemos exclusivamente por las alusiones que a ella hacen Aristóteles, Cicerón y Quintiliano y cuya figura se encuentra indisolublemente unida a la de su discípulo Tisias, como tendremos ocasión de ver más adelante. Cicerón, citando un texto perdido de Aristóteles, señala que:

"Nadie, antes de él, había acostumbrado litigar con método y técnica, aun cuando la mayor parte de ellos lo hacía con pericia y precisión" (46).

En términos generales, y por lo que podemos saber a partir de las referencias existentes, el manual consistía en un conjunto de técnicas argumentativo-retóricas destinadas al desempeño exitoso en el contexto público de los tribunales. Sin lugar a dudas, su principal contribución se encuentra en el reconocimiento de cuatro partes del discurso y sus fines.

Tras el fin del régimen de los Tiranos (en donde muchas de las tierras de los ciudadanos habían sido confiscadas y repartidas entre los miembros del ejército) hubo lugar para un gran número de pleitos de tierras (presentados como alegato público ante asamblea de jueces y ciudadanos reunida en el ágora) que buscaban reivindicar el

\footnotetext{
${ }^{6}$ Murphy parece apoyar esta opinión aludiendo a los abundantes fragmentos argumentativos contenidos en La Ilíada y en piezas dramáticas como Las Euménides de Esquilo. Véase: Murphy, J., 1989, Pp. 9-12.

7 "Corax" en algunos textos. De él no sabemos mucho, ya que su obra no ha llegado íntegra hasta nuestros días, se sabe, sin embargo, que vivió en Siracusa y que, posteriormente al período de los Tiranos, se dedicó a componer discursos para los reclamantes en los pleitos de tierra suscitados tras la caída de este régimen.
} 
derecho de posesión de sus dueños originales y, en tal contexto, Korax se hizo notorio como logógrafo, es decir, como un compositor de discursos para los distintos litigantes, oficio por el que recibía una conveniente remuneración. Tanto Breton y Gauthier $(2000,13)$, como otros autores (aunque de manera menos esquemática), coinciden en atribuirle el establecimiento de un primer esquema de construcción del discurso argumentativo que, reconociendo cuatro partes del mismo, puede presentarse del modo siguiente:

\begin{tabular}{|c|c|c|c|}
\hline EXORDIO & $\begin{array}{c}\text { PRESENTACIÓN DE } \\
\text { HECHOS }\end{array}$ & DISCUSIÓN & PERORACIÓN \\
\hline $\begin{array}{c}\text { Captar la atención del } \\
\text { auditorio. }\end{array}$ & $\begin{array}{c}\text { Decir de qué se habla, } \\
\text { exponer la propia tesis. }\end{array}$ & $\begin{array}{c}\text { Proporcionar los } \\
\text { argumentos a favor de } \\
\text { la tesis. }\end{array}$ & $\begin{array}{c}\text { Terminar por una } \\
\text { fórmula sintética. }\end{array}$ \\
\hline
\end{tabular}

En donde es posible notar, además de una muy cercana semejanza con los esquemas que Aristóteles ofrece en su Retórica, la presencia de un conjunto de objetivos identificadores de las acciones que es preciso implementar para la instanciación de cada una de las partes. El esquema parece haber tenido un gran éxito y haber sido rápidamente adoptado por otros logógrafos, entre estos, evidentemente, se encuentran los discípulos indirectos de Korax por medio de las enseñanzas de Tisias. En efecto, resulta notorio el hecho de que Dionisio de Halicarnaso, en su magnífico examen de los retores antiguos, cimenta buena parte de sus juicios en el modo en que cada uno de estos retores construye cada uno de los distintos momentos del discurso y, así, subraya en Lisias $^{8}$ que maneja con particular maestría la construcción del exordio, indicando las diversas estrategias que utiliza para iniciarlo y que se resumen en:

- Comenzar con un elogio a sí mismo (bastante velado).

- Acusar directamente al adversario.

- Si es acusado, destruir en primer lugar las imputaciones que se le dirigen.

- Alabar a los jueces para hacerlos favorables a su persona y a su causa.

- Presentar la cuestión que trata como de interés general, de alta importancia y que merece toda la atención del auditorio.

- Oponer su debilidad al prestigio y fuerza de su adversario para subrayar la desigualdad de la lucha que va a emprender (Gros, 62-65).

\footnotetext{
${ }^{8} 458$ - 380 a. de C. Nacido en Atenas, nunca llegó a gozar de nacionalidad ática debido a que era hijo de un siracusano y a que, cuando este privilegio le fue concedido muchos años después, no pudo ejercerlo por alguna formalidad no seguida en el momento en que la asamblea se lo otorgó, Cicerón parece conocer este antecedente y le reivindica la nacionalidad ática porque "certe Athenis est et natus et mortuus et functus omni civium munere (ciertamente nació y murió en Atenas y cumplió con todo deber de ciudadano).
} 
Del mismo modo, indica que Iseo " "pone la narración (presentación de los hechos) en el lugar más conveniente, le da poca extensión y no incluye en ella ninguna prueba" (Gros, 288), y agrega que es Isócrates ${ }^{10}$ el verdadero maestro en la presentación, encadenamiento y distribución de las pruebas (172). Citas, todas estas, que claramente demuestran la temprana y exitosa asunción de los preceptos korácicos en materia de estructura $u$ orden del discurso argumentativo.

Sin embargo, aunque las referencias al manual de Korax que aún sobreviven muestran con meridiana claridad el desarrollo de una técnica oratoria, la verdad es que el estatus de "arte" de esta ocupación fue largo tiempo discutido sin que parezca haberse presentado una solución explícita hasta la aparición de Lisias, quien, a decir de Cicerón, fue el primero en afirmar la existencia de este "oficio de orador"11.

Nam Lysiam primo profiteri splitum artem esse dicendi: Lisias, pues, solía declarar que había arte del decir (Cicerón, Brutus, 17).

b) El establecimiento de la Antifonía como espacio natural de ocurrencia del discurso argumentativo

Plantin llama Antífona a "la práctica sistemática de contraponer discursos"(8), indicando además que "Todo argumento puede volverse del revés, y a todo discurso le responde un contradiscurso producido desde otro punto de vista y proyectando una realidad diferente"(8), sin embargo, desde nuestra perspectiva, en su planteamiento hay dos aspectos distintos aunque complementarios: la legitimación de la posibilidad de contraargumentar, derecho connatural a todo locutor constituido en argumentador en una instancia de discusión y, también, el carácter esencialmente dialógico del intercambio argumentativo y que se relaciona con la posibilidad de intercambiar los turnos de habla entre los distintos participantes en este intercambio. Es el segundo de estos sentidos el que consideramos adecuado para la identificación del aporte de esta etapa a las teorías de la argumentación, ya que supone la instalación de un precepto que, de acuerdo con Murphy, entronca con un momento de evolución del Ditirambo ${ }^{12}$, en donde "dos partes opuestas o antitéticas del coro ditirámbico" (Murphy, 11) se separaron dando origen a la forma de diálogo que reconocemos ya en la tragedia griega del siglo VII a. de C. y que, evidentemente, constituye el espacio natural de ocurrencia del discurso argumentativo, pues, ha de tenerse en cuenta que los discursos construidos según los precedentemente señalados "preceptos korácicos" tenían como destino su ejecución en el ágora, un espacio en el que el diálogo,

\footnotetext{
${ }^{9} 420-340$ a. de C.

${ }^{10} 436-338$ a. de C.

${ }^{11}$ Cuestión muy debatida y relevante porque la respuesta afirmativa a la misma confería legitimidad a la enseñanza de la oratoria, pero, al mismo tiempo, le exigía la exhibición de los procedimientos y la casuística de aplicación de los mismos que era requisito y rasgo identitario de todo oficio en la época.

12 Composición destinada a ser cantada y/o bailada por un coro de 50 personas durante las representaciones públicas, sin un líder.
} 
sometido ya a algún tipo de pautamiento semejante al que hoy observamos en el juicio oral del Sistema Procesal Penal, no dejaba por ello de ser muy dinámico, como lo delineaba Vernant (1962) en los siguientes términos:

"forma el centro de un espacio público en común. Todos los que entran en él se definen, por lo mismo, como iguales [...] por su presencia en este espacio político, entran en relaciones de perfecta reciprocidad los unos con los otros" (126).

Y, respecto de los procesos que tenían lugar allí, apuntaba Barthes (1970) que:

"Estos procesos eran de un tipo nuevo: movilizaban grandes jurados populares, delante de los cuales, para convencer, era preciso ser 'elocuente'. Esta elocuencia, que participaba al mismo tiempo de la democracia y de la demagogia, de lo judicial y de lo político, se constituyó rápidamente en objeto de enseñanza" (175, traducción nuestra).

En lo que nosotros vemos, además de la explicación del hecho de que la crítica antigua dedique muchas páginas al "estilo" de los distintos retores, la instalación de un sistema de toma y cesión de turnos de habla que, antecediendo en muchos siglos al moderno juicio oral, es responsable del alto pautamiento que hoy exhibe esta forma de interacción argumentativa.

c) El establecimiento de la Dialéctica como procedimiento

En estrecha relación con lo anterior, la Dialéctica es un procedimiento que tiene lugar en el marco del diálogo, pero en un nivel de especificación mayor, toda vez que el procedimiento se centra en el aspecto razonado o razonable del diálogo en cuestión, es decir, en su contenido. Al respecto, Plantin señala que "la palabra 'dialéctica' tiene múltiples significados [...] se aplica aquí, y en los estudios de la argumentación en general, a una forma de diálogo razonado, conducido según reglas precisas" $(10)$, indicación que, a nuestro juicio, se vincula más naturalmente con el primero de los sentidos apuntados en el acápite anterior, es decir, con la idea de que la discusión racional de un hecho que, porque no resulta practicable o evidente, exige la presentación de argumentos y contraargumentos que permitan discernir su estatus de real, cuestión que se dirimirá, en definitiva, por el valor de mayor o menor verdad que ofrezcan los argumentos presentados por las partes, aunque en realidad se trate más bien de mayor o menor "verosimilitud", como apuntará Aristóteles un tiempo después.

Este aspecto, evidentemente, se relaciona estrechamente con los "tipos de argumento" utilizables en la discusión y esto, a su vez, con la finalidad persuasiva o convincente de la misma; distinción, esta última, que no parece haber sido explícitamente hecha en la época que nos ocupa y que, de acuerdo con nuestro propio 
rastreo de la misma, aparece por primera vez en el propio Dionisio de Halicarnaso ${ }^{13}$, quien, refiriéndose a Lisias, contrapone las formas verbales $\pi \imath \sigma \alpha v \alpha \iota$ y $\pi \imath \sigma \tau \nu^{14} \mathrm{y}$, aunque la distinción entre estas dos categorías podría resultar oscura en el pasaje en el que aparece, resulta aclarada por el hecho de que el propio Dionisio organiza su

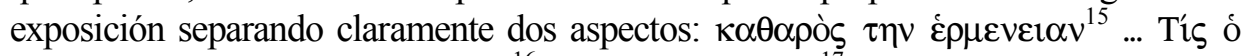

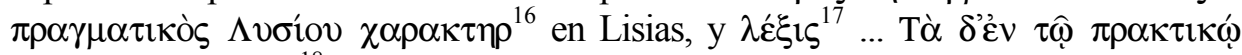

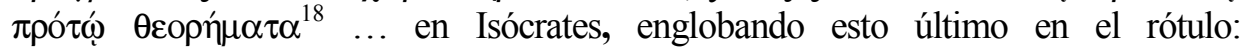

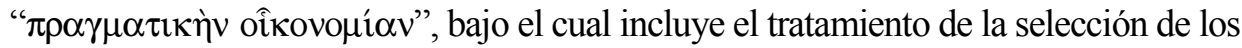
argumentos. Respecto de los tipos de argumento, a su vez, tampoco contamos con un elenco de los mismos en esta época, pero Dionisio subraya la capacidad de Lisias para presentar pruebas acordes a la cuestión que trata, mediante un certero uso del ejemplo $^{19}$ y un cuidado por hacer manifiesto en qué se parece al tema que trata y en qué difiere de él, logrando, finalmente, "transformar en pruebas ciertas las simples probabilidades" (70). Todo ello nos lleva a pensar en que el "ejemplo", forma argumental que explicará e instanciará Aristóteles un siglo más tarde, era ya parte del elenco argumental de la época a la que nos referimos.

\section{d) La legitimación de la Paradoja como posibilidad contraargumentativa}

La paradoja es, en el sentido de la Real Academia, una "figura del pensamiento que consiste en emplear expresiones o frases que envuelven contradicción" y también: "idea extraña u opuesta a la común opinión o al sentir de las personas"; es decir, su rasgo más característico es la contradicción y, por ello, supone la aparición (en el contexto argumentativo) de una posibilidad no prevista y que, no obstante, adecuadamente argumentada, puede llegar a constituirse en una forma válida de resolución de una disputa. En relación con esto, Plantin indica que la virtud de la paradoja consiste en hacer evidente la autonomía, chocante para el 'sentido común', de los funcionamientos lingüísticos en relación con la realidad.

e) El establecimiento de la Probabilidad como forma argumental

Tanto Breton y Gauthier (13) como Murphy (15) coinciden en atribuir a Korax la autoría de la Probabilidad general como forma argumental válida. El contexto, por supuesto, es el que hemos descrito más arriba: el de la Siracusa post gobierno de los Tiranos en la que se ha establecido como costumbre que las disputas sean resueltas

\footnotetext{
${ }^{13}$ Recuérdese que vivió mucho tiempo después de los autores que trata (60 -7 a. de C.).

${ }^{14}$ Dionisio de Halicarnaso en GROS, E., op cit. pp. 69.

15 "La pureza de su estilo".

${ }^{16}$ Su "manera" de relación de las cosas.

${ }^{17}$ Estilo.

${ }^{18}$ Acerca de la relación de las cosas.

${ }^{19}$ Recuérdese que Aristóteles recomienda el uso argumentativo del ejemplo y el entimema como formas privilegiadas de argumento, aunque se inclina principalmente por el último.
} 
con alegato público ante un grupo de jueces, la diferencia es que ahora no se trata de un litigio por la posesión de la tierra, sino de una demanda que el propio maestro Korax entabla contra su discípulo más dilecto, Tisias, exigiéndole que pague el importe por las enseñanzas recibidas. El antecedente de esta disputa es la formulación de la probabilidad en el siguiente contexto: un hombre robusto reclama haber sido golpeado por un hombre menudo, de manera que el alegato discurre del modo siguiente:

$\begin{array}{ll}\text { HOMBRE MENUDO } \quad \text { : } & \text { (Acusado de golpear a un hombre corpulento) "No es } \\ & \text { probable que yo hiciera tal cosa, porque el hombre } \\ & \text { corpulento es más fuerte que yo y me vencería, y, como } \\ & \text { yo lo sé, no iba a provocar su ira golpeándolo". }\end{array}$

Podrá notarse aquí que nos encontramos ante una disyuntiva muy difícil de resolver, toda vez que no existe ninguna evidencia "practicable" (asequible) de los hechos y, en tal caso, ambos argumentos parecen aceptables en tanto que razonables y esto es, precisamente, lo que pretende el argumento de probabilidad que deja a los aspectos retóricos (estilísticos) del discurso la carga de obtener la persuasión o convicción de los jueces. Pues bien, Korax utiliza este mismo tipo de argumento para apoyar su demanda de pago contra su discípulo Tisias, pero el discípulo ha aprendido muy bien la lección, de manera que se defiende con el mismo argumento, con lo que la confrontación asume la siguiente forma.
KORAX : "Debes PAGARME si ganas el caso porque eso demostraría el valor de mis lecciones. Si pierdes el caso, debes pagarme también porque el tribunal te obligará a hacerlo. Tanto en una situación como en la otra, debes pagarme".
TISIAS : "No pagaré nada, porque, si pierdo el caso, quedaría demostrado que tus enseñanzas carecían de valor. Por otro lado, si gano, el tribunal me absolverá de pagar. En una situación u otra, no pagaré".

Por supuesto, el final de esta historia no es claro, Murphy señala que "el tribunal aplazó la sentencia indefinidamente" (15) y Plantin indica que "los jueces, ante esta aporía, persiguieron a los litigantes a bastonazos" (7). Sea cual sea el final, lo cierto es que la contribución que Korax hace a este período del desarrollo de las teorías de la argumentación y al aspecto concreto que presentamos aquí, es la incorporación de un tipo específico de argumento, cuya resolución dependerá, como ya lo hemos dicho, de la cobertura estilística en la que se lo presente, hecho que, de paso, explica el énfasis retórico que esta época parece subrayar. 
Finalmente, la construcción de estos cimientos para el ulterior desarrollo de las teorías de la argumentación es tributario de la reflexión y esfuerzo de unos más o menos "oscuros" autores que dieron forma al período que nos ocupa y a quienes debemos, además, la institucionalización de la enseñanza de la "Técnica Oratoria", no podemos cerrar esta comunicación sin nombrarlos. Partimos, obviamente, por la mención del maestro Korax de Siracusa (S. V a. de C.) y de su discípulo Tisias, del que no sabemos mucho, pero que la tradición clásica sitúa como maestro de Lisias (458-380 a. de C.) e Isócrates (436-338 a. de C.); Protágoras (485-411 a. de C.), Gorgias (485-380 a. de C.), Iseo (420-340 a. de C.) y Dinarco (361-291 a. de C.). Todos ellos contribuyeron a constituir buena parte de la preceptiva argumental que hoy conocemos y, sobre todo, a hacer de la argumentación un oficio legítimo digno de ser enseñado como instrumento privilegiado de resolución "incruenta" de las diferencias de opinión en la vida cotidiana, razón fundamental por la que se le ha resucitado en contexto de guerra fría y por la que se le incluye hoy en el currículum escolar. Abrigamos la esperanza de dedicar un examen más exhaustivo a cada uno de ellos en ulteriores aportaciones.

\section{CONCLUSIONES}

Este sucinto examen de los principales problemas vinculados a la construcción de una historia de las teorías de la argumentación manifiesta los hechos siguientes:

La cuestión de la periodización del desarrollo de las teorías de la argumentación continuará siendo un tema abierto hasta que no se convenga en un criterio general para establecer los cortes diacrónicos que corresponde hacer. Hasta aquí, parece haber primado un criterio que vincula el ejercicio argumentativo con una específica forma de gobierno (la democracia) y su uso crea un vacío histórico de más de un milenio en el que, no obstante, resulta de sentido común pensar que sí ha habido algún tipo de desarrollo que todavía queda por investigar.

Respecto de la cuestión de la conceptualización, es factible aceptar que, aunque difusa en los albores clásicos, la distinción entre retórica y argumentación (como dominios de conocimientos separados y complementarios) se justifica ya en la propia tradición clásica, como podemos reconocerlo en los propios textos de Dionisio de Halicarnaso y que, por lo tanto, su reunificación no puede ser buscada en la continuación de esa tradición, sino en su integración en una comprensión interactiva del fenómeno argumentativo, para ello el tratamiento pragmalingüístico puede constituir un camino exitoso. Y otro tanto podemos afirmar de la distinción entre finalidad persuasiva y convincente del discurso argumentativo.

La cuestión de los índices descriptores del texto argumentativo, a su vez y aun cuando constituye una tarea pendiente de los estudios pragmalingüísticos, puede resolverse medianamente aun con los "bastones" teóricos que todavía suministra la tradición clásica, aun cuando estos son susceptibles de ser enriquecidos con su integración en las contemporáneas teorías de la interacción. 
El período fundacional de las Teorías de la Argumentación, aun cuando ha sido tan escuetamente presentado en esta comunicación, manifiesta una época extraordinariamente prolífica que ha marcado fuertemente el desarrollo subsiguiente de las Teorías de la Argumentación, pero cuya significancia ha resultado obnubilada por el velo de prejuicio que la tradición de la reflexión socrática (y platónica) ha superpuesto sobre la sofística.

Es de esperar, esperanza que anima nuestra investigación, que ulteriores estudios relativos a los textos producidos por los autores de esta época logren conferir a la sofística el legítimo estatus que le corresponde en el origen de las Teorías de la Argumentación.

\author{
Universidad de Los Lagos* \\ Departamento de Humanidades y Arte \\ Avda. Alcalde Fuchslocher 1305, Osorno (Chile) \\ erisco@ulagos.cl
}

\title{
OBRAS CITADAS
}

Barthes, Roland. "L'ancienne rhétorique". En: Communications, recherches rhétoriques' 16, Paris, Seuil, 1970.

Breton, Philippe y Gauthier, Gilles. Histoire des théories de l'argumentation. Paris: La découverte, 2000.

Cicerón, Marco Tulio. Bruto. De los Oradores Ilustres. Introducción, traducción y notas de Bulmaro Reyes Coria. México: Universidad Nacional Autónoma de México, 2004.

Gros, E. Examen critique del plus célèbres écrivains de la Grèce, par Denys d'Halicarnasse. Paris: Brunot-Labbe éditeur. 1826. Tome Prémier.

Murphy, James. Sinopsis histórica de la retórica clásica. Madrid: Gredos, 1989.

Pantin, Christian. La Argumentación. Barcelona: Ariel, 2002

Vernant, J-P. Les origines de la pensé grecque. París: P.U.F, 1962. 